OPEN ACCESS

Edited by:

Xihui Shen,

Northwest A\&F University, China

Reviewed by:

Laura R. Jarboe,

lowa State University, United States

Feng Jiang,

Chinese Academy of Medical

Sciences, China

Yi-Cheng Sun,

Institute of Pathogen Biology (CAMS),

China

*Correspondence:

Xiaoyun Liu

xiaoyun.liu@pku.edu.cn

tThese authors have contributed

equally to this work

Specialty section: This article was submitted to

Infectious Diseases,

a section of the journa

Frontiers in Microbiology

Received: 14 June 2018 Accepted: 14 August 2018 Published: 31 August 2018

Citation:

Zhang B, Ran L, Wu M, Li Z, Jiang J, Wang Z, Cheng S, Fu J and Liu X (2018) Shigella flexneri Regulator SlyA Controls Bacterial Acid Resistance by Directly Activating the Glutamate

Decarboxylation System.

Front. Microbiol. 9:2071.

doi: 10.3389/fmicb.2018.02071

\section{Shigella flexneri Regulator SlyA Controls Bacterial Acid Resistance by Directly Activating the Glutamate Decarboxylation System}

\author{
Buyu Zhang', Longhao Ran', Mei Wu, Zezhou Li, Jiezhang Jiang, Zhen Wang, \\ Sen Cheng, Jiaqi Fu and Xiaoyun Liu*
}

Institute of Analytical Chemistry and Synthetic and Functional Biomolecules Center, College of Chemistry and Molecular

Engineering, Peking University, Beijing, China

Shigella flexneri is an important foodborne bacterial pathogen with infectious dose as low as 10-100 cells. SlyA, a transcriptional regulator of the MarR family, has been shown to regulate virulence in a closely related bacterial pathogen, Salmonella Typhimurium. However, the regulatory role of SlyA in S. flexneri is less understood. Here we applied unbiased proteomic profiling to define the SlyA regulon in S. flexneri. We found that the genetic ablation of slyA led to the alteration of 18 bacterial proteins among over 1400 identifications. Intriguingly, most down-regulated proteins (whose expression is SlyA-dependent) were associated with bacterial acid resistance such as the glutamate decarboxylation system. We further demonstrated that SlyA directly regulates the expression of GadA, a glutamate decarboxylase, by binding to the promotor region of its coding gene. Importantly, overexpression of GadA was able to rescue the survival defect of the $\triangle$ slyA mutant under acid stress. Therefore, our study highlights a major role of SlyA in controlling S. flexneri acid resistance and provides a molecular mechanism underlying such regulation as well.

Keywords: Shigella flexneri, SlyA, acid resistance, glutamate decarboxylation, GadA

\section{INTRODUCTION}

The bacterium Shigella flexneri (S. flexneri) is an intracellular pathogen that causes gut infection resulting in watery diarrhea or bacillary dysentery (Kotloff et al., 1999). It is estimated that there are over 160 million cases of shigellosis per year worldwide, leading to more than half a million deaths mostly associated with infants in developing countries (Kotloff et al., 1999). A major virulence determinant of $S$. flexneri is a large 230-kb plasmid that encodes the bacterial type III secretion system (T3SS) (Mattock and Blocker, 2017). Working as a molecular syringe, the T3SS is able to translocate an extensive repertoire of effector proteins into host cells, promoting initial bacterial internalization as well as intracellular replication. As a foodborne pathogen, S. flexneri infection of the gastrointestinal (GI) tract requires successful passage and survival through the highly acidic stomach. A rather striking feature of this bacterium is its extremely low infectious dose of as few as 10-100 bacterial cells. Therefore, bacterial acid resistance, in addition to its T3SS, constitutes another critical virulence determinant in Shigella spp.

Bacteria such as S. flexneri have evolved a plethora of strategies to cope with acid stress. A classical anti-acid mechanism of great importance is the amino acid (e.g., glutamate) 
decarboxylase-dependent systems (Jennison and Verma, 2007). In principle, protons are consumed by glutamate decarboxylation together with the release of $\mathrm{CO}_{2}$, thereby raising the cytoplasmic $\mathrm{pH}$. GadA and GadB are the central enzymes in this process that catalyze the decarboxylating reaction (Bearson et al., 1997). Over the years, this important anti-acid mechanism has been well characterized (Hersh et al., 1996; De Biase et al., 1999; Castanie-Cornet and Foster, 2001; Foster, 2004); however, its transcriptional regulation still remains largely unclear in S. flexneri.

The MarR/SlyA family of transcription factors regulates a variety of bacterial cellular processes (George and Levy, 1983; Roper et al., 1993; Thomson et al., 1997; Srikumar et al., 1998; Galan et al., 2003). In Salmonella Typhimurium, for instance, SlyA has been shown to be an important regulator of bacterial virulence. The slyA deletion mutant exhibits a survival defect within macrophages as well as in a mouse model of infection (Libby et al., 1994; Buchmeier et al., 1997). Further transcriptome studies of the slyA deletion mutant in comparison to the wildtype strain revealed that it controls the expression of several known virulence genes (Navarre et al., 2005). In addition, many of the SlyA-controlled genes are also regulated by the PhoPPhoQ two-component system (Navarre et al., 2005). In contrast, the regulatory role of SlyA in S. flexneri is less characterized thus far. In a pioneering study, Weatherspoon-Griffin and Wing (2016) recently reported the association of overexpressed SlyA with $S$. flexneri virulence. They also uncovered a survival defect of the slyA deletion mutant under acid stress, though the underlying mechanisms remain unclear (Weatherspoon-Griffin and Wing, 2016).

Here we performed unbiased proteomic profiling of a S. flexneri mutant lacking slyA in comparison to its parental strain. We found differential regulation of 18 proteins among over 1400 detected bacterial proteins. Notably, our data revealed SlyA-dependent expression of GadA and GadB (the glutamate decarboxylase), suggesting a direct link of this transcription factor to $S$. flexneri acid resistance. We provided further evidence that SlyA regulates the expression of Gad proteins by directly binding to the gene promoter regions. Importantly, $S$. flexneri mutants lacking slyA exhibited a severe survival defect under acid challenge and overproduction of the glutamate decarboxylase was able to restore, to a large extent, the bacterial anti-acid capacity of the sly $A$ deletion mutant. Therefore, our study provides the first paradigm in bacteria that SlyA controls acid resistance by directly activating the glutamate decarboxylation system.

\section{MATERIALS AND METHODS}

\section{Bacterial Strains and Construction of Mutants}

The S. flexneri serotype 2a 2457T (generously provided by Dr. Feng Shao from National Institute of Biological Sciences, Beijing, China) was used in this study, and the bacteria were routinely grown on trypticase soy agar plates with $1.5 \%$ agar and $30 \mu \mathrm{g} / \mathrm{mL}$ streptomycin at $37^{\circ} \mathrm{C}$. A single colony picked from the plates was inoculated into trypticase soy broth (TSB) with $30 \mu \mathrm{g} / \mathrm{mL}$ streptomycin, and then the overnight culture was diluted 1:20 into $3 \mathrm{~mL}$ of TSB. The bacteria were harvested at indicated $\mathrm{OD}_{600}$ for different assays at $4^{\circ} \mathrm{C}$. The sly $A$ deletion mutant $(\triangle s l y A)$ was constructed using the homologous recombination method as previously described (Liu et al., 2015). Successful deletion of a target gene was confirmed by both PCR analyses and sequencing. To construct the complementation strains harboring a plasmid-borne $s l y A$ or gadA gene in the $\triangle s l y A$ background ( $\Delta s l y A+$ pSlyA or $\Delta s l y A+\mathrm{pGadA}$ ), the $s l y A$ or gadA fragment was amplified, digested, and inserted into the pME6032 plasmid with an isopropyl $\beta$-D-1-thiogalactopyranoside (IPTG)inducible promoter and a C-terminal $3 \times$ FLAG tag. For $\beta$-galactosidase assays, the upstream region (1000 bp) of the gadA gene containing the promoter region was cloned and inserted into the pNN387 plasmid which contains a promoterless lacZ (Elledge and Davis, 1989). All primers and strains used in this study are listed in the Supplementary Table S1.

\section{Sample Preparation, Protein Digestion, and Stable Isotope Dimethyl Labeling}

To uncover potential SlyA-regulated proteins, we performed proteomic analyses of the $\Delta s l y A$ strain together with the wildtype (WT) strain. In brief, bacterial proteins were digested and isotopically labeled prior to two-dimensional LC-MS/MS analyses. Bacterial subculture was grown to an $\mathrm{OD}_{600}$ of $\sim 0.9$ and pelleted at $3000 \times g$ for $5 \mathrm{~min}$. Bacterial pellets were washed with ice-cold PBS ( $\mathrm{pH}=7.5$ ) and resuspended in $1 \mathrm{~mL}$ of $8 \mathrm{M}$ urea with $50 \mathrm{mM} \mathrm{NH} \mathrm{NHCO}_{3}$. Then bacterial cells were lysed by sonication and protein concentration was measured by using a BCA protein assay kit (Solarbio, China). After diluted to one fourth with $50 \mathrm{mM} \mathrm{NH} \mathrm{HCO}_{3}, 1 \mathrm{mg}$ of proteins from each sample was digested with trypsin (Promega, United States) overnight at an enzyme-to-protein ratio of $1: 50(\mathrm{w} / \mathrm{w})$ at $37^{\circ} \mathrm{C}$. Resulting tryptic peptides were desalted and labeled on C18 Sep-Pak cartridges (Welch, China). For desalting, the columns were activated with $1 \mathrm{~mL}$ of acetonitrile (ACN) containing $0.1 \%$ (vol/vol) formic acid (FA) and equilibrated with $1 \mathrm{~mL}$ of $0.1 \%$ FA. Then the resulting tryptic peptides were loaded onto the columns. After washing with $1 \mathrm{~mL}$ of $0.1 \% \mathrm{FA}$, the on-column isotopic labeling experiments were performed as described previously (Boersema et al., 2009). Peptides on the column were repeatedly treated for three times by $1 \mathrm{~mL}$ of the labeling solution containing $100 \mathrm{mM}$ triethyl ammonium bicarbonate (TEAB), $30 \mathrm{mM}$ sodium cyanoborohydride $\left(\mathrm{NaBH}_{3} \mathrm{CN}\right), 0.2 \%$ formaldehyde $\left(\mathrm{CH}_{2} \mathrm{O}\right)$ or deuterated formaldehyde $\left(\mathrm{CD}_{2} \mathrm{O}\right)$. Peptides from WT and $\Delta s l y A$ strains were labeled with $\mathrm{CH}_{2} \mathrm{O}$ and $\mathrm{CD}_{2} \mathrm{O}$, respectively. After labeling, the peptides were washed once with $0.1 \% \mathrm{FA}$ and eluted with $70 \% \mathrm{ACN}$ plus $0.1 \% \mathrm{FA}$. Then light- and heavy-labeled peptides were pooled and vacuum dried for further offline fractionation.

\section{Offline High pH Reversed-Phase Fractionation and Nanoflow LC-MS/MS Analyses}

Peptide samples were further fractionated using C18 SepPak cartridges (Welch, China) under high $\mathrm{pH}$ conditions 
(10 $\mathrm{mM} \mathrm{NH}_{4} \mathrm{HCO}_{3}, \mathrm{pH}$ 10). For peptide elution, the following percentages of ACN were used: $6,9,12,15,18,21,25,30$, and $35 \%$ (Lai et al., 2016). In total, nine fractions were collected and pooled into six samples $(6+25 \%, 9+30 \%, 12+35 \%, 15 \%, 18 \%$, and $21 \%$ ). Samples were vacuum dried for subsequent LC-MS analyses. Nanoflow reversed-phase LC separation was carried out on an EASY-nLC 1200 system (Thermo Fisher Scientific). The capillary column $(75 \mu \mathrm{m} \times 150 \mathrm{~mm})$ with a laser-pulled electrospray tip (Model P-2000, Sutter Instruments) was homepacked with $4 \mu \mathrm{m}, 100 \AA$ Magic C18AQ silica-based particles (Michrom BioResources Inc., Auburn, CA, United States). The mobile phase was composed of Solvent A $\left(97 \% \mathrm{H}_{2} \mathrm{O}, 3 \% \mathrm{ACN}\right.$, and $0.1 \% \mathrm{FA})$ and Solvent $\mathrm{B}\left(20 \% \mathrm{H}_{2} \mathrm{O}, 80 \% \mathrm{ACN}\right.$, and $\left.0.1 \% \mathrm{FA}\right)$. The LC separation was carried out at room temperature with the following gradient: Solvent B was started at $7 \%$ for $3 \mathrm{~min}$, and then raised to $40 \%$ over 120 min; subsequently, Solvent B was rapidly increased to $90 \%$ in $2 \mathrm{~min}$ and maintained for $10 \mathrm{~min}$ before $100 \%$ Solvent A was used for column equilibration. Eluted peptides from the capillary column were electrosprayed directly into a hybrid linear ion trap-Orbitrap mass spectrometer (LTQ Orbitrap Velos, Thermo Fisher Scientific) for MS and MS/MS analyses in a data-dependent acquisition mode. One full MS scan $(m / z$ 350-1200) was acquired and then MS/MS analyses were performed on the 10 most intense ions. The selected ions were fragmented by collision-induced dissociation (CID) in the ion trap with the following parameters: $\geq+2$ precursor ion charge, $2 \mathrm{Da}$ precursor ion isolation window, and 35\% normalized collision energy. Dynamic exclusion was set with repeat duration of $24 \mathrm{~s}$ and exclusion duration of $12 \mathrm{~s}$.

\section{Proteomic Data Processing}

Raw MS files were processed by MaxQuant (version 1.5.4.1) and searched against the S. flexneri (strain 301/serotype 2a) protein database (4103 sequences, downloaded from UniProt). The precursor mass tolerance was set at $20 \mathrm{ppm}$ and the fragment mass tolerance was set at $0.8 \mathrm{Da}$. Maximum missed cleavage was set to 2. Dimethyl (K, N-term) and dimethyl $\left(\mathrm{D}_{4} \mathrm{~K}, \mathrm{D}_{4} \mathrm{~N}\right.$-term) were set as variable modifications for light $(\mathrm{L})$ - and heavy $(\mathrm{H})$ labeled samples, respectively. Oxidation (M) was set as a variable modification. Both peptide and protein assignments were filtered to achieve a false discovery rate (FDR) $<1 \%$. Only proteins with at least two unique plus razor peptides were quantified. The protein group lists were further processed by using the Perseus software (version 1.5.4.1) for the calculation of logarithmic values $\left(\log _{2}\right)$ of the $\mathrm{H}$ - and L-labeled protein intensity ratio and the $p$-values. Proteins with ratios $(\mathrm{H} / \mathrm{L})>2.0$ or $<0.5$ and $p$-values $<0.05$ were considered as significant difference between the WT and $\Delta s l y A$ strains.

\section{Quantitative Real-Time PCR}

Both the WT and $\Delta s l y A$ strains were grown to an $\mathrm{OD}_{600}$ of $\sim 0.9$ as described above. Total bacterial RNA from $1 \mathrm{~mL}$ of culture was extracted by using an EasyPure RNA Kit (TransGen Biotech, China) and then treated with DNase I. Reverse transcription of RNA was performed with TransScript One-Step gDNA Removal and cDNA Synthesis SuperMix (TransGen Biotech, China). RTPCR analyses were carried out on an Applied Biosystems ViiA ${ }^{\mathrm{TM}}$
7 Real-Time PCR System by using UltraSYBR Mixture (Low ROX) (CWBIO, China). To quantitatively compare the levels of gadA, hdeB, slp, and SF2991 transcripts, the housekeeping $16 \mathrm{~S}$ rRNA gene was used for normalization. The mRNA levels were determined using the comparative threshold cycle number $\left(2^{-\Delta \Delta C_{\mathrm{t}}}\right)$ method (Livak and Schmitge, 2001).

\section{$\beta$-Galactosidase Assays}

Shigella cells were grown in TSB at $37^{\circ} \mathrm{C}$ to an $\mathrm{OD}_{600}$ of $\sim 1.0$. Bacterial cells from $1.2 \mathrm{~mL}$ of culture were pelleted at $14,000 \times g$ for $2 \mathrm{~min}$ and then resuspended in $1.2 \mathrm{~mL}$ of $\mathrm{Z}$ buffer $(60 \mathrm{mM}$ $\mathrm{Na}_{2} \mathrm{HPO}_{4}, 40 \mathrm{mM} \mathrm{NaH} \mathrm{PO}_{4}, 10 \mathrm{mM} \mathrm{KCl}$, and $1 \mathrm{mM} \mathrm{MgSO}_{4}$ ) plus $50 \mathrm{mM} \beta$-mercaptoethanol (freshly added). Subsequently, $30 \mu \mathrm{L}$ of chloroform and $15 \mu \mathrm{L}$ of $0.1 \%$ SDS were added and mixed upon vortexing. The assays were started by the addition of $240 \mu \mathrm{L}$ of $4 \mathrm{mg} / \mathrm{mL}$ o-nitrophenyl- $\beta$-D-galactopyranoside (ONPG). Upon the observation of a faint yellow color, the reaction was quenched by the addition of $600 \mu \mathrm{L}$ of $1 \mathrm{M}$ $\mathrm{Na}_{2} \mathrm{CO}_{3}$ and the reaction time was recorded. Finally, samples were centrifuged at $14,000 \times g$ for $2 \mathrm{~min}$, and the $\mathrm{OD}_{420}$ of the supernatant was recorded. Assay units were calculated as $1,000 \times \mathrm{OD}_{420} /\left(\mathrm{OD}_{600}\right)$ (total reaction time) .

\section{Expression and Purification of Recombinant Proteins}

His $_{6}$-tagged SlyA was expressed in the E. coli strain BL21 (DE3) harboring the appropriate plasmid. Briefly, $15 \mathrm{~mL}$ of the overnight culture was added in $300 \mathrm{~mL}$ LB broth and grown until the $\mathrm{OD}_{600}$ reached $0.6-0.8$. Upon the addition of IPTG to a final concentration of $0.2 \mathrm{mM}$, bacterial culture was incubated further in a shaker at $18^{\circ} \mathrm{C}$ for $16-18 \mathrm{~h}$. Bacterial cells (from $300 \mathrm{~mL}$ of culture) were lysed in $30 \mathrm{~mL}$ of ice cold PBS buffer via sonication and cell lysates were clarified by centrifugation at 5,000 $\times g$ for $10 \mathrm{~min}$ three times at $4^{\circ} \mathrm{C}$. His-tagged proteins were captured with Ni-NTA Resin (GenScript) and washed serially with PBS and $20 \mathrm{mM}$ imidazole. Finally, proteins were eluted with $300 \mathrm{mM}$ imidazole. Purified proteins were further dialyzed overnight in $25 \mathrm{mM}$ Tris- $\mathrm{HCl}$ (pH 7.5), $150 \mathrm{mM} \mathrm{NaCl}, 5 \%$ (vol/vol) glycerol, and $1 \mathrm{mM}$ DTT with at least two buffer changes.

\section{Electrophoretic Mobility Shift Assays (EMSAs)}

The putative promoter sequence of $\operatorname{gadA}$, a mutated version in which the SlyA-binding sequence (TTATCATGTTAA) was deleted and a DNA fragment of its coding sequence were amplified by PCR, purified with a Gel Extraction Kit (TransGen Biotech, China) and dissolved in water. Then purified SlyA proteins were incubated with $40 \mathrm{nM}$ DNA fragments in $20 \mu \mathrm{L}$ of binding buffer (10 mM Tris- $\mathrm{HCl}$ ( $\mathrm{pH} 7.5), 100 \mathrm{mM} \mathrm{KCl}$, $1 \mathrm{mM}$ EDTA, $0.1 \mathrm{mM}$ DTT, 5\% v/v glycerol, and $10 \mu \mathrm{g} / \mathrm{mL}$ BSA). Molar ratios between DNA fragment and SlyA were set at $1: 0,1: 30,1: 35,1: 40,1: 45$, and 1:50 (indicated as $0,1.2,1.4,1.6$, 1.8, and 2.0 $\mu \mathrm{M}$ SlyA, respectively, in Figure 3 ). The reaction mixtures were incubated at room temperature for $30 \mathrm{~min}$ and then loaded onto $8 \%$ native polyacrylamide gels. Electrophoresis was performed using $0.5 \times$ TBE buffer $(44.5 \mathrm{mM}$ Tris, $44.5 \mathrm{mM}$ 
boric acid, and $1 \mathrm{mM}$ EDTA) in ice bath. The gel was stained with Gel Stain (Yeasen, China) and photographed by using a Tanon-1600 Gel Image System (Tanon, China).

\section{Bacterial Acid Survival Assays}

The overnight cultures of individual Shigella strains were diluted 1:20 into $3 \mathrm{~mL}$ of LB. When $\mathrm{OD}_{600}$ reached about 0.6 , IPTG was added to a final concentration of $0.2 \mathrm{mM}$ to induce the expression of plasmid-borne SlyA or GadA for $4 \mathrm{~h}$. Then bacterial cells were diluted 1:50 in the acidified LB media ( $\mathrm{pH} 2.5$ adjusted with $\mathrm{HCl}$ ). Viable cell counts were determined at 0,1 , and $2 \mathrm{~h}$ post acid challenge by serial dilution and plating on LB agar plates. At least three repetitions were performed for each experiment.

\section{RESULTS}

\section{Comparative Proteomic Profiling of S. flexneri Wild-Type and Its Isogenic $\Delta$ slyA Strains}

To study the SlyA regulon in S. flexneri, we carried out quantitative proteomic profiling of a bacterial strain lacking $\operatorname{sly} \mathrm{A}$ $(\triangle s l y A)$ and its parental wild-type (WT) strain. In total, we identified $1410 \mathrm{~S}$. flexneri proteins from three biological replicates (i.e., six bacterial samples). A complete list of all identified bacterial proteins is provided in Supplementary Table S2. To get a global view of differentially expressed proteins, we plotted the ratio of protein abundance $(\Delta s l y A / \mathrm{WT})$ as a function of calculated $p$-values (Figure 1). As seen in the protein-level volcano plot, the two proteomes are strikingly similar with vast majority of proteins lining up toward the center, indicating comparable expression levels of most proteins between two bacterial strains. By using the criteria described in the "Materials and Methods" section, 18 proteins were differentially regulated in the $s l y A$-lacking strain, including 5 up-regulated and 13 downregulated proteins (see Table 1). The most significant hit in the up-regulated proteins is SF2991/S3195, which is annotated as an outer membrane fluffing protein. The exact function of this protein has yet to be characterized. With respect to the downregulated proteins, notably several proteins are well-known mediators of bacterial acid resistance such as GadA, GadB, GadC, and HdeB. The glutamate decarboxylation pathway represents arguably the most prominent example of bacterial anti-acid mechanisms. GadA and GadB are two glutamate decarboxylases catalyzing the proton-consuming reaction. MurI is a racemase catalyzing the interconversion of L-glutamate to D-glutamate, and therefore it is related to the GadAB-mediated bacterial antiacid system.

Furthermore, some of the other proteins that exhibited lower expression levels in the $\triangle s l y A$ strain seem to be associated with bacterial acid resistance as well. For instance, YdeP was previously reported to confer acid resistance in E. coli (Masuda and Church, 2003). Interestingly, its regulator EvgA also appears in the list of those down-regulated proteins. In addition, the homologs of Frc and S2577 (i.e., YfdW and YfdU, respectively) were shown to play a role in E. coli acid resistance through a

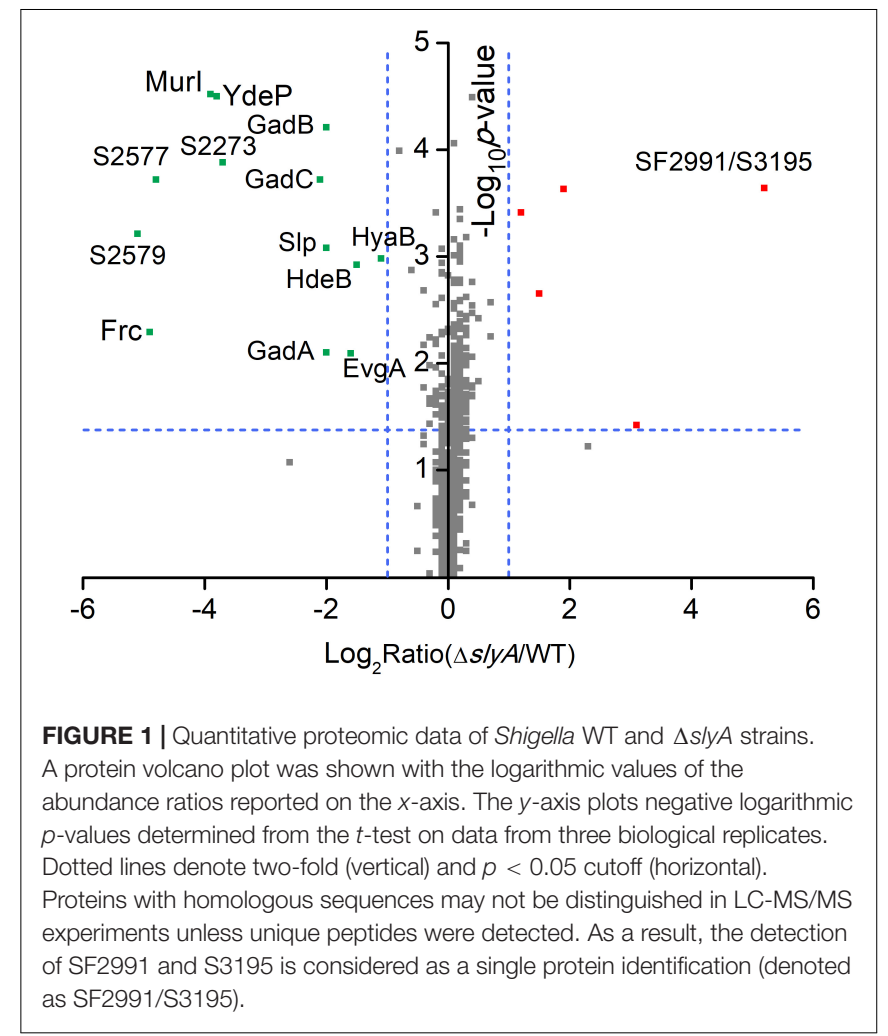

mechanism involving oxalate decarboxylation (Fontenot et al., 2013). Another protein, Slp, is an outer membrane protein that was depressed in the $\triangle$ slyA strain, consistent with a previous study of SlyA-regulated proteins in E. coli (Spory et al., 2002). Interestingly, we previously found that $S$. flexneri up-regulates Slp in response to acid stress (Yu et al., 2017). Taken together, these findings strongly suggest SlyA-dependent expression of many Shigella proteins associated with bacterial anti-acid mechanisms. Next we focused our attention on the characterization of the regulatory role of SlyA in mediating S. flexneri anti-acid capacities.

\section{Transcriptional Regulation of S. flexneri Anti-acid Genes by SlyA}

Proteomic observations of SlyA-dependent production of those anti-acid proteins prompted us to determine if SlyA, as a transcription factor, exerts its regulatory role on the transcript level. We carried out qRT-PCR measurements on some representative genes whose protein products were differentially expressed in the $\Delta s l y A$ strain including $g a d A, h d e B$, slp, and SF2991 (Figure 2A). Consistent with the down-regulation of their corresponding proteins, the mRNA levels of $g a d A, h d e B$, and $s l p$ were profoundly lower in the slyA-deletion mutant than those in its parental strain. Additionally, the transcript of SF2991, a SlyArepressed gene, was found to be substantially higher ( $\sim 60$-fold) in the mutant strain. Unlike gadA and $s l p$, the fold difference of $h d e B$ and SF2991 transcript levels is markedly larger than that of their protein levels between the $\Delta s l y A$ and WT strains, indicating potential mechanisms of post-transcriptional regulation. 
TABLE 1 | A list of altered Shigella proteins in the $\triangle$ slyA mutant.

\begin{tabular}{|c|c|c|c|c|}
\hline & Gene & Protein description & Ratio & $p$-Value \\
\hline \multirow[t]{5}{*}{ Up-regulated } & SF2991/S3195 & Outer membrane fluffing protein & 36.7 & 0.0002 \\
\hline & $S 1803$ & Putative membrane protein & 8.5 & 0.0376 \\
\hline & metE & 5-Methyltetrahydropteroyltriglutamate-homocysteine methyltransferase & 3.7 & 0.0002 \\
\hline & metF & 5,10-Methylenetetrahydrofolate reductase & 2.8 & 0.0022 \\
\hline & yaeC & Lipoprotein & 2.4 & 0.0004 \\
\hline \multirow[t]{13}{*}{ Down-regulated } & S2579 & Uncharacterized protein & 0.0 & 0.0006 \\
\hline & fre & Formyl-coenzyme A transferase & 0.0 & 0.0051 \\
\hline & S2577 & Putative enzyme & 0.0 & 0.0002 \\
\hline & murl & Glutamate racemase & 0.1 & 0.0000 \\
\hline & $y d e P$ & Protein YdeP & 0.1 & 0.0000 \\
\hline & S2273 & Uncharacterized protein & 0.1 & 0.0001 \\
\hline & $s / p$ & Outer membrane protein induced after carbon starvation & 0.2 & 0.0002 \\
\hline & $\operatorname{gad} B$ & Glutamate decarboxylase beta & 0.2 & 0.0001 \\
\hline & $\operatorname{gad} A$ & Glutamate decarboxylase alpha & 0.2 & 0.0079 \\
\hline & gadC & Glutamate/gamma-aminobutyrate antiporter & 0.3 & 0.0008 \\
\hline & $\operatorname{evgA}$ & Positive transcription regulator & 0.3 & 0.0082 \\
\hline & $h d e B$ & Acid stress chaperone HdeB & 0.3 & 0.0012 \\
\hline & hyaB & Hydrogenase- 1 large chain & 0.5 & 0.0011 \\
\hline
\end{tabular}

Given the prominent role of the glutamate decarboxylation system in acid resistance, next we further explored the transcriptional control of gadA by SlyA by utilizing a $\beta$-galactosidase-based reporter assay. We constructed a lacZ-expressing plasmid in which its promoter region was replaced by that of gadA and transformed this plasmid into Shigella strains of different genetic backgrounds (WT, $\triangle$ slyA, and $\triangle$ sly $A+$ pSlyA). By measuring the enzymatic activities of expressed $\beta$-galactosidase in vitro, we could assess the promoter activity of the gadA gene in different strains. As shown in Figure $2 \mathrm{~B}, \beta$-galactosidase activity decreased substantially (by at least an order of magnitude) in the $\Delta s l y A$ mutant relative to that in its parental strain, indicating that the promoter activity of gadA is strongly dependent on SlyA. Furthermore, ectopic expression of SlyA in the $\Delta s l y A$ mutant largely restored the promoter activity to the wild-type level. Taken together, these results established that SlyA positively regulates the expression of $\operatorname{gadA}$ on the transcript level and likely other S. flexneri genes involved in acid resistance as well.

\section{Direct Binding of S. flexneri SlyA to the Promoter Region of the gadA Gene}

SlyA is a transcriptional regulator in the MarR family with a classical helix-turn-helix (HTH) DNA-binding domain (Ellison and Miller, 2006). Next we sought to determine if SlyA exerts a direct regulatory role in GadA expression by using electrophoretic mobility shift assays (EMSAs). Recombinantly purified SlyA proteins were incubated with various concentrations of DNA fragments spanning the gadA promoter region (the promoter region and putative SlyA-binding site are shown in Figure 3A). Electrophoretic experiments demonstrated that with increasing levels of SlyA a greater fraction of DNA fragments exhibited a mobility shift (Figure 3B). In contrast, when DNA fragments containing a partial sequence of $\mathrm{gadA}$-coding region were used as a negative control, we did not observe any retardation in electrophoretic mobility at all concentrations of SlyA. Remarkably, deletion of the putative SlyA-binding site in the gadA promoter sequence completely abolished its binding to SlyA (Figure 3B). Together, these findings reveal that SlyA directly controls the expression of GadA by physically associating with the gene promoter region.

\section{S. flexneri SlyA Mediates Acid Resistance by Regulating the Expression of Gad Proteins}

Given its direct regulatory role in the glutamate decarboxylation system, we hypothesized that SlyA may modulate Shigella acid resistance. To test our hypothesis, we challenged the $\Delta$ slyA strain in highly acidic conditions ( $\mathrm{pH} 2.5)$ for different durations ( 1 and $2 \mathrm{~h}$ ) and then determined bacterial viability by CFU assays (Figure 4). Compared to its parental WT strain, upon 1 and $2 \mathrm{~h}$ of acid challenge the slyA-deletion mutant exhibited substantially lower survival percentages ( $>50$-fold and 300 -fold less, respectively). Furthermore, we were able to rescue such a survival defect by reintroducing a functional copy of slyA into the $\Delta s l y A$ strain $(\Delta s l y A+$ pSlyA). Next, we sought out to determine if the regulation of bacterial acid resistance by SlyA is largely accomplished by its control of the glutamate decarboxylation pathway (e.g., the expression of GadA). To explore this possibility, we further constructed a bacterial strain harboring a copy of plasmid-borne gadA in the $\Delta s l y A$ background. Remarkably, ectopic expression of GadA can restore, to a large extent, bacterial acid resistance of the $\Delta s l y A$ strain to the levels of the complementation strain $(\Delta s l y A+\mathrm{pSlyA})$ as well as the WT bacteria. Nonetheless, we do note a minor difference in the rescuing ability of two complementation strains as suggested by the slightly lower (yet significant) survival 


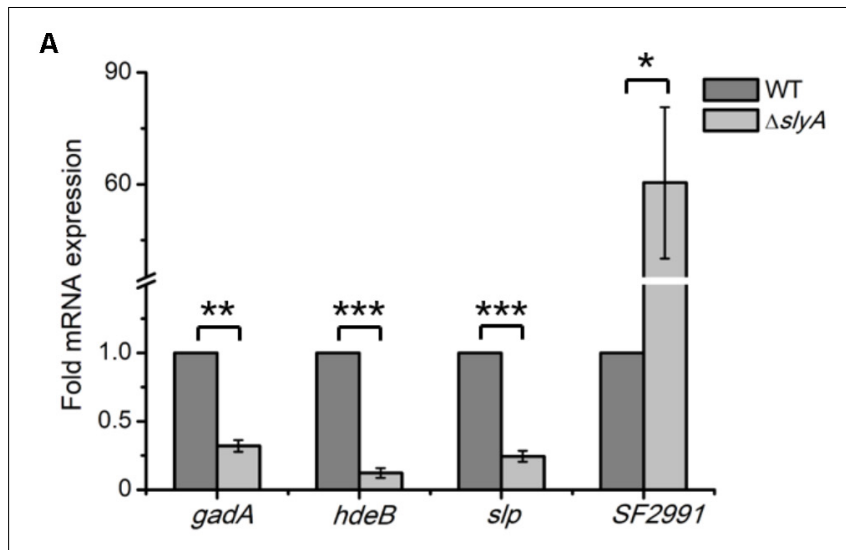

8

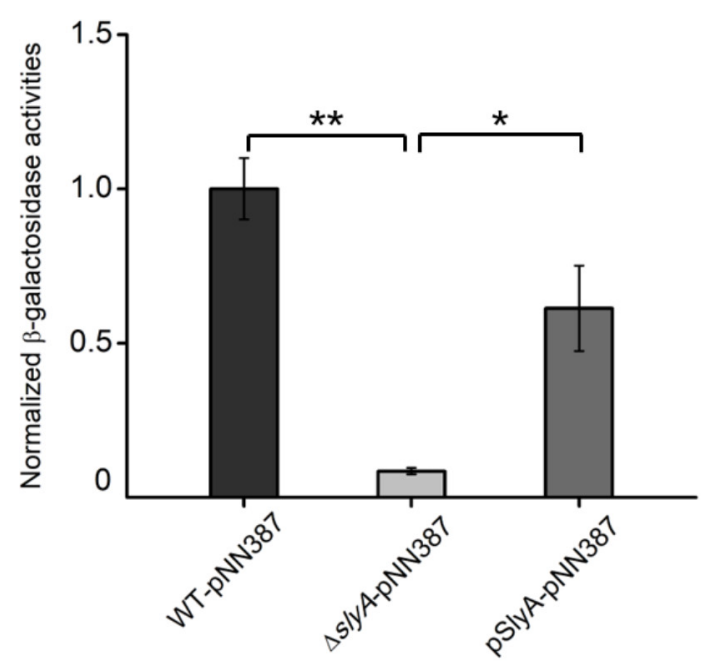

FIGURE 2 | SlyA activates the expression of gadA at the transcriptional level. (A) qRT-PCR analyses of mRNA samples extracted from Shigella WT and $\triangle$ slyA strains. (B) Measurements of $\beta$-galactosidase activities in WT, $\Delta$ slyA, and $\triangle$ slyA+pSlyA strains harboring a plasmid encoding a promoterless lac $Z$ fused with the gadA promoter. $\beta$-Galactosidase activities from three biological replicates are shown with values normalized to that of the WT strain. Asterisks indicate significant differences $\left({ }^{*} p<0.05,{ }^{* *} p<0.01\right.$, and $\left.{ }^{* * *} p<0.001\right)$.

percentage of the $\triangle s l y A$ strain ectopically producing GadA than the SlyA-complemented mutant. Taken together, these data suggest Shigella SlyA plays a crucial role in bacterial acid resistance and importantly such regulation is mostly exerted through its control of the glutamate decarboxylation pathway.

\section{DISCUSSION}

The MarR/SlyA family of transcriptional regulators has been identified in a variety of Gram-negative bacteria. In Salmonella Typhimurium, SlyA was reported to contribute to bacterial virulence by exerting a regulatory effect on SsrB (Navarre et al., 2005), a master regulator of Salmonella pathogenicity island 2 (SPI-2). Consistent with this notion, our previous work

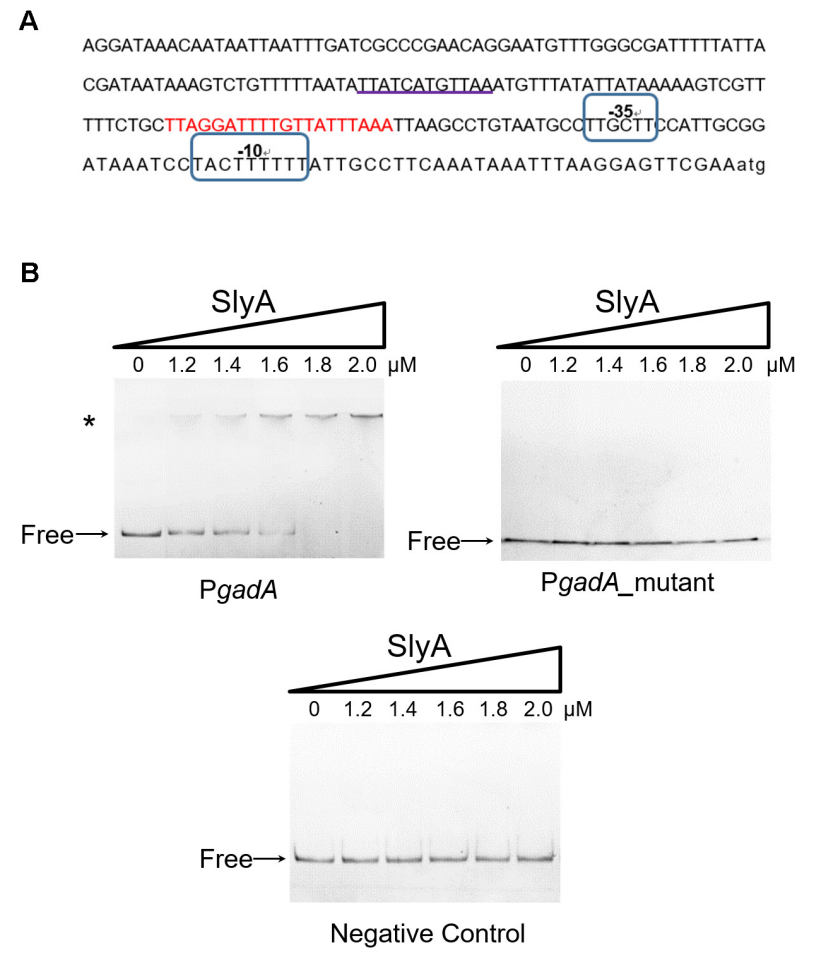

FIGURE 3 | SlyA directly binds to the promoter region of the gadA gene. (A) Bioinformatics analysis of the gadA promoter region (PgadA). The sequence of the gadA promoter region of $224 \mathrm{bp}$ upstream of the start codon is shown and the putative SlyA-binding site is underlined. Putative -10 and -35 elements are circled and the gad box is indicated in red. (B) EMSA experiments determined the direct binding of SlyA to PgadA. Different concentrations of purified SlyA (ranging from 0 to $2 \mu \mathrm{M}$ ) were incubated with PgadA, PgadA lacking the SlyA-binding site (PgadA_mutant) or negative control DNA prior to electrophoretic separation. "Free" indicates free DNA and the asterisk indicates DNA-protein complexes.

showed concurrent induction of S. Typhimurium SlyA and SPI2-encoded virulence proteins during infection of epithelial cells (Liu et al., 2015, 2017). In addition to virulence, SlyA has been associated with other aspects of $S$. Typhimurium physiology including resistance to antimicrobial peptides (Navarre et al., 2005) and oxidative stress (Buchmeier et al., 1997). Unlike in $S$. Typhimurium, the regulatory role of SlyA in $S$. flexneri has been less defined. Previously, Weatherspoon-Griffin and Wing (2016) carried out the initial characterization of S. flexneri SlyA and identified a role in bacterial virulence as well. Our current study aims to examine the SlyA regulon in S. flexneri by using an unbiased proteomic approach. Quantitative profiling of a $S$. flexneri mutant lacking sly $A$ in comparison to its parental strain led to the discovery of SlyA-dependent expression of many proteins associated with acid resistance such as GadA/B, HdeB, and Slp. Interestingly, the implication of SlyA in bacterial antiacid defense was reported in Wing's study, though the exact mechanism was not clarified (Weatherspoon-Griffin and Wing, 2016).

Among many bacterial anti-acid strategies, the glutamate decarboxylase-dependent pathway is one of the most effective 


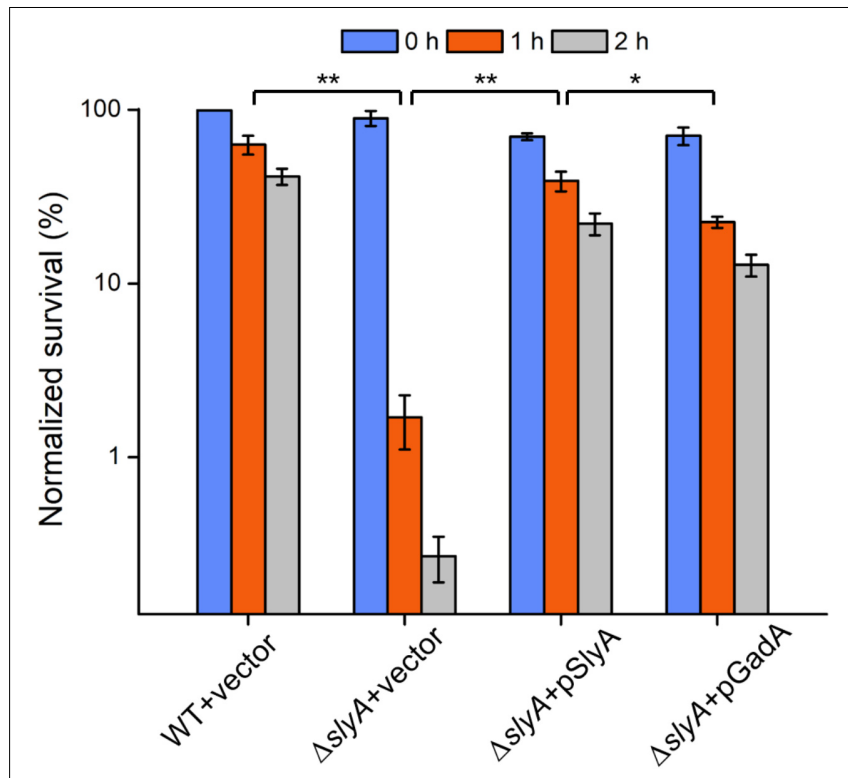

FIGURE 4 | Acid survival assays of various Shigella strains. Survival percentages of Shigella wild-type and $\Delta$ slyA strains complemented with either empty vectors or SlyA/GadA-expressing plasmids were shown. Bacteria were incubated in acidic conditions for 0,1 , and $2 \mathrm{~h}$ and assayed for viability. Asterisks indicate significant differences $\left({ }^{*} p<0.05\right.$ and $\left.{ }^{* *} p<0.01\right)$.

systems for acid resistance. This system consists of three proteins, GadA, GadB, and GadC, all of which were downregulated in the slyA deletion mutant. We further verified SlyAdependent transcription of $\operatorname{gadA}$ by both mRNA measurements and $\beta$-galactosidase-based reporter assays. Importantly, we found SlyA directly regulates the expression of $\operatorname{gadA}$ by binding to its promoter region. After establishing the expression of GadA (and likely the entire Gad system) in a SlyA-dependent manner, we further examined the anti-acid capacities of the slyA-deletion mutant. Consistent with the previous report, the strain lacking $\operatorname{sly} A$ exhibited a severe survival defect upon acid challenge. Complementation with a SlyA-expressing plasmid was able to restore its anti-acid capacities to the level of the wild-type bacteria. If the glutamate decarboxylasedependent pathway constitutes the major anti-acid system under the control of SlyA, we reasoned that the introduction of a GadA-expressing plasmid would rescue, at least in part, the survival defect of the slyA-deletion mutant under acid stress. Indeed, we found overproduction of GadA in the slyAdeletion strain largely restored its anti-acid capacities, though at slightly lower levels than the $\Delta s l y A$ strain harboring a SlyAproducing plasmid as well as the WT bacteria. Such observations are in fact consistent with our proteomic data suggesting SlyA-dependent activation of multiple anti-acid pathways in addition to the Gad system. In other words, SlyA may regulate S. flexneri acid resistance through downstream proteins other than GadA.

Shigella flexneri gadB and gadC genes are located in a transcriptional unit different from that of $\operatorname{gadA}$. In E. coli, a number of regulators have been identified to control the transcription of these gad genes, such as GadE ( $\mathrm{Ma}$ et al., 2003a), GadW (Tucker et al., 2003), GadX (Tucker et al., 2003), PhoP (Zwir et al., 2005), YdeO (Ma et al., 2004), EvgA (Masuda and Church, 2003; Ma et al., 2004), RpoS (De Biase et al., 1999), and Crp (Ma et al., 2003b). Notably, GadE plays a central role in transcriptional regulation and activates the expression of gad genes by directly binding to the gad box (shown in Figure 3A), a 20-bp sequence located 63-bp upstream of the transcriptional start sites of $\mathrm{gadA} / B$
A SlyA_binding_sequence.seq PgadA_-80 -200bp.seq Consensus

SlyA_binding_sequence.seq PgadA_-80 -200bp.seq Consensus

SlyA_binding_sequence.seq PgadA_-80 -200bp.seq Consensus

B SlyA_binding_sequence.seq PgadE_-160 -280bp.seq Consensus

SlyA binding_sequence.seq PgadE_-160 -280bp.seq Consensus

SlyA_binding_sequence.seq Pgad̄E_-160 -280bp.seq Consensus
CGCCCGAACAGGAAT GTTT GGGCGATTTTTATTACGATAA $\quad \stackrel{0}{40}$

TTAGCAAGOTAA
TAAAGT CT GTTTTTAATATTATCATGTTAAAT GTTATAT

$\mathrm{tta} c a \mathrm{~g} \mathrm{taa}$

TATAAAAAGT CGTTTTTCTCCTTAGGATTTTTTTATTTAA 12

20

ACAAACT GGTTATT GATAACTTATTCTT GEGCAGTAATCC $\quad 40$

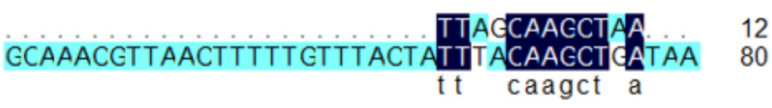

CAACCAGGAAT CTTACTTAGGAT CAATATATGGAGT GCGT 120

FIGURE 5 | Alignment analyses revealed consensus SlyA-binding sequence in the promoter regions of the gadA and gadE genes. The alignment of consensus SlyA-binding sequence and fragments of the promoter of gadA $(\mathbf{A})$ and gadE $(\mathbf{B})$ was performed by the DNAMAN software. 


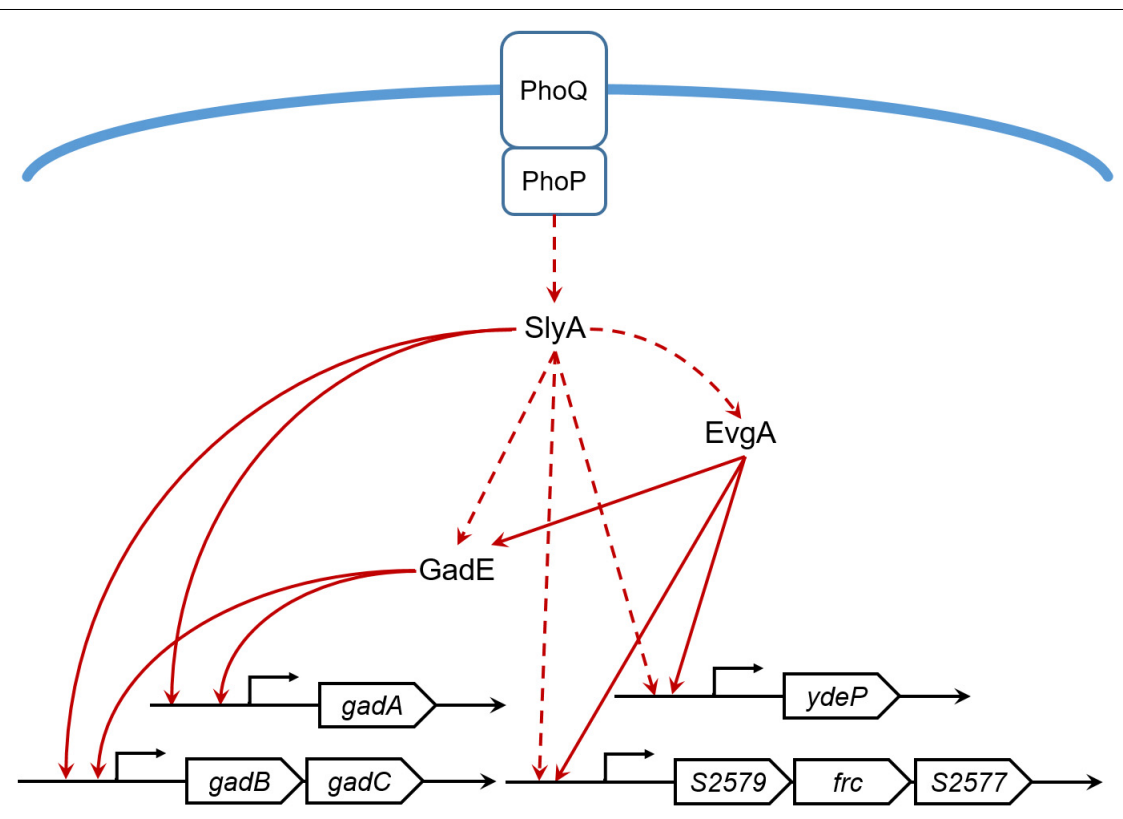

FIGURE 6 | The SlyA regulatory network of S. flexneri acid resistance. Solid lines indicate direct or experimentally verified transcriptional regulation. Dashed lines indicate hypothetical regulation inferred from our proteomic results or literatures.

and gadC (Castanie-Cornet and Foster, 2001). Most of the other transcription factors above were found to regulate the expression of Gad proteins via the control of GadE. For instance, two AraC-like regulators, GadW and GadX, whose genes are located downstream of $\mathrm{gadA}$, directly activate the transcription of $\mathrm{gadE}$ and hence the expression of $g a d A / B$ and $g a d C$ (Ma et al., 2002; Tramonti et al., 2002, 2003). However, under certain conditions, GadW and GadX can function as repressors by competitively binding to the gad box sequences (Castanie-Cornet and Foster, 2001; Ma et al., 2002; Tramonti et al., 2002). Thus, our discovery that S. flexneri SlyA regulates the transcription of gadA by directly binding to its promoter further expands the repertoire of regulators controlling the glutamate decarboxylase-dependent system. Previously, E. coli SlyA was also reported to regulate the expression of GadA and other proteins involved in acid resistance such as HdeA and HdeB (Spory et al., 2002). It is therefore tempting to speculate that the regulation of acid resistance by SlyA may be a conserved mechanism shared by many bacterial species.

Our proteomic data also revealed other SlyA-regulated proteins involved in acid resistance such as EvgA, YdeP, Frc, and S2577. EvgA is a member of the two-component regulatory system EvgS/EvgA, which activates the transcription of other regulators such as $\mathrm{YdeO}$ and GadE, thereby leading to increased expression of the Gad system (Ma et al., 2004). YdeP is a putative oxidoreductase under the control of EvgA. Intriguingly, overexpression of this enzyme in E. coli enhanced bacterial acid resistance (Masuda and Church, 2003). Frc and S2577 are homologous to the formyl-CoA transferase YfdW and the oxalyl-CoA decarboxylase YfdU in E. coli, respectively. Previously Fontenot et al. (2013) reported the contribution of these two enzymes to bacterial anti-acid capacities involving oxalate decarboxylation. Interestingly, the yfdXWUVE operon is also controlled by EvgA (Masuda and Church, 2002), and $y f d X$ encodes a protein homologous to S2579, another down-regulated protein in our dataset. Given that EvgA regulates Frc, S2577, and S2579 in addition to the Gad system, it would be tempting to determine whether SlyA exerts a regulatory role in EvgA and thus indirectly influences the expression of the $y f d$ operon. Furthermore, by using the $\mathrm{gadA}$ promoter as a positive control, the alignment of the SlyA-binding consensus (Stapleton et al., 2002) and the gadE gene sequence suggests direct binding of SlyA to the gadE promoter region (Figure 5). Considering the complicated regulatory network, we propose that SlyA might be a global transcriptional regulator of Shigella acid resistance (Figure 6), though experimental validation of SlyA-exerted regulatory roles in other known mediators of acid resistance would be highly desired in the future. Furthermore, given the direct binding of PhoP to the SlyA promoter in Salmonella Typhimurium (Shi et al., 2004), it is conceivable that SlyA is activated by other transcriptional factors such as PhoP rather than direct sensing of the environmental $\mathrm{pH}$. Indeed, Shigella slyA promoter was also reported to respond to $\mathrm{Mg}^{2+}$ and be positively regulated by PhoP (Weatherspoon-Griffin and Wing, 2016).

As a final note, we found insignificant difference in most T3SSrelated proteins in the $s l y A$ deletion mutant (Supplementary Table S3), although Weatherspoon-Griffin and Wing (2016) suggested a potential role of SlyA in mediating S. flexneri virulence. In their previous work, ectopic expression of SlyA was found to increase the promoter activity of certain virulence genes and also rescue a virulence-associated phenotype. We reason that such observations may be resulted from the overproduction 
of SlyA from a high-copy-number plasmid, thereby differing to some extent from our proteomic measurements in which SlyA was expressed at physiological levels. Therefore, we propose that SlyA is likely to be a global transcriptional regulator of bacterial acid resistance in S. flexneri, which is in contrast to virulence regulation in $S$. Typhimurium.

\section{DATA AVAILABILITY}

The proteomics data reported in this paper have been deposited to the iProX database (URL: http://www.iprox.org/page/ HMV006.html) under the accession number IPX0001250001.

\section{AUTHOR CONTRIBUTIONS}

$\mathrm{BZ}$ and LR conducted most of the experiments with the assistance from MW, ZL, JJ, ZW, and SC. JF, BZ, LR, and XL wrote the manuscript with editorial inputs from all authors.

\section{FUNDING}

This work was financially supported by grants from the National Natural Science Foundation of China (21475005 and 21622501),

\section{REFERENCES}

Bearson, S., Bearson, B., and Foster, J. W. (1997). Acid stress responses in enterobacteria. FEMS Microbiol. Lett. 147, 173-180. doi: 10.1111/j.1574-6968. 1997

Boersema, P. J., Raijmakers, R., Lemeer, S., Mohammed, S., and Heck, A. J. (2009). Multiplex peptide stable isotope dimethyl labeling for quantitative proteomics. Nat. Protoc. 4, 484-494. doi: 10.1038/nprot.2009.21

Buchmeier, N., Bossie, S., Chen, C. Y., Fang, F. C., Guiney, D. G., and Libby, S. J. (1997). SlyA, a transcriptional regulator of Salmonella typhimurium, is required for resistance to oxidative stress and is expressed in the intracellular environment of macrophages. Infect. Immun. 65, 3725-3730.

Castanie-Cornet, M. P., and Foster, J. W. (2001). Escherichia coli acid resistance: cAMP receptor protein and a $20 \mathrm{bp}$ cis-acting sequence control $\mathrm{pH}$ and stationary phase expression of the gadA and gadBC glutamate decarboxylase genes. Microbiology 147, 709-715. doi: 10.1099/00221287-1473-709

De Biase, D., Tramonti, A., Bossa, F., and Visca, P. (1999). The response to stationary-phase stress conditions in Escherichia coli role and regulation of the glutamic acid decarboxylase system. Mol. Microbiol. 32, 1198-1211. doi: 10.1046/j.1365-2958.1999.01430.x

Elledge, S. J., and Davis, R. W. (1989). Position and density effects on repression by stationary and mobile DNA-binding proteins. Genes Dev. 3, 185-197. doi: $10.1101 / \operatorname{gad} .3 .2 .185$

Ellison, D. W., and Miller, V. L. (2006). Regulation of virulence by members of the MarR SlyA family. Curr. Opin. Microbiol. 9, 153-159. doi: 10.1016/j.mib.2006. 02.003

Fontenot, E. M., Ezelle, K. E., Gabreski, L. N., Giglio, E. R., McAfee, J. M., and Mills, A. C. et al. (2013). YfdW and YfdU are required for oxalate-induced acid tolerance in Escherichia coli K-12. J. Bacteriol. 195, 1446-1455. doi: 10.1128/JB. 01936-1912.

Foster, J. W. (2004). Escherichia coli acid resistance tales of an amateur acidophile. Nat. Rev. Microbiol. 2, 898-907. doi: 10.1038/nrmicro1021

Galan, B., Kolb, A., Sanz, J. M., Garcia, J. L., and Prieto, M. A. (2003). Molecular determinants of the $h p a$ regulatory system of Escherichia coli: the HpaR repressor. Nucleic Acids Res. 31, 6598-6609. doi: 10.1093/nar/gkg851
Clinical Medicine Plus X-Young Scholars Project of Peking University, and the Thousand Young Talents Program of the Chinese government.

\section{ACKNOWLEDGMENTS}

We thank the members of the Liu laboratory for careful review of this manuscript. We also thank Dr. Guifang Jia for the use of the Applied Biosystems ViiA ${ }^{\mathrm{TM}} 7$ Real-Time PCR System and Dr. Aixin Yan for the kind gift of the plasmid pNN387.

\section{SUPPLEMENTARY MATERIAL}

The Supplementary Material for this article can be found online at: https://www.frontiersin.org/articles/10.3389/fmicb. 2018.02071/full\#supplementary-material

TABLE S1 | All strains and primers used in this study.

TABLE S2 | All identified bacterial proteins.

TABLE S3 | Virulence proteins identified in the proteomic profiling

George, A. M., and Levy, S. B. (1983). Gene in the major cotransduction gap of the Escherichia coli K-12 linkage map required for the expression of chromosomal resistance to tetracycline and other antibiotics. J. Bacteriol. 155, 541-548.

Hersh, B. M., Farooq, F. T., Barstad, D. N., Blankenhorn, D. L., and Slonczewski, J. L. (1996). A glutamate-dependent acid resistance gene in Escherichia coli. J. Bacteriol. 178, 3978-3981. doi: 10.1128/jb.178.13.3978-3981.1996

Jennison, A. V., and Verma, N. K. (2007). The acid-resistance pathways of Shigella flexneri 2457T. Microbiology 153, 2593-2602. doi: 10.1099/mic.0.2007/ 006718-0

Kotloff, K. L., Winickoff, J. P., Ivanoff, B., Clemens, J. D., Swerdlow, D. L., and Sansonetti, P. J. et al. (1999). Global burden of Shigella infections implications for vaccine development and implementation of control strategies. Bull. World Health Organ. 77, 651-666.

Lai, M., Liang, L., Chen, J., Qiu, N., Ge, S., and Ji, S. et al. (2016). Multidimensional proteomics reveals a role of UHRF2 in the regulation of epithelial-mesenchymal transition (EMT). Mol. Cell. Proteomics 15, 2263-2278. doi: 10.1074/mcp.M115. 057448

Libby, S. J., Goebel, W., Ludwig, A., Buchmeier, N., Bowe, F., and Fang, F. C. et al (1994). A cytolysin encoded by Salmonella is required for survival within macrophages. Proc. Natl. Acad. Sci. U.S.A. 91, 489-493. doi: 10.1073/pnas.91. 2.489

Liu, Y., Yu, K., Zhou, F., Ding, T., Yang, Y., and Hu, M. (2017). Quantitative proteomics charts the landscape of Salmonella carbon metabolism within host epithelial cells. J. Proteome Res. 16, 788-797. doi: 10.1021/acs.jproteome. $6 \mathrm{~b} 00793$

Liu, Y., Zhang, Q., Hu, M., Yu, K., Fu, J., and Zhou, F. et al. (2015). Proteomic analyses of intracellular Salmonella enterica serovar Typhimurium reveal extensive bacterial adaptations to infected host epithelial cells. Infect. Immun. 83, 2897-2906. doi: 10.1128/IAI.02882-14

Livak, K. J., and Schmitge, T. D. (2001). Analysis of relative gene expression data using real-time quantitative PCR and the $2^{-\Delta \Delta C}{ }_{T}$ method. Methods 25, 402-408. doi: 10.1006/meth.2001.1262

Ma, Z., Gong, S., Richard, H., Tucker, D. L., Conway, T., and Foster, J. W. (2003a). GadE (YhiE) activates glutamate decarboxylase-dependent acid resistance in Escherichia coli K-12. Mol. Microbiol. 49, 1309-1320. 
Ma, Z., Richard, H., and Foster, J. W. (2003b). pH-Dependent modulation of cyclic AMP levels and GadW-dependent repression of RpoS affect synthesis of the GadX regulator and Escherichia coli acid resistance. J. Bacteriol. 185, 6852-6859.

Ma, Z., Masuda, N., and Foster, J. W. (2004). Characterization of EvgASYdeO-GadE branched regulatory circuit governing glutamate-dependent acid resistance in Escherichia coli. J. Bacteriol. 186, 7378-7389. doi: 10.1128/JB.186. 21.7378-7389.2004

Ma, Z., Richard, H., Tucker, D. L., Conway, T., and Foster, J. W. (2002). Collaborative regulation of Escherichia coli glutamate-dependent acid resistance by two AraC-like regulators, GadX and GadW (YhiW). J. Bacteriol. 184, 7001-7012. doi: 10.1128/JB.184.24.7001-7012.2002

Masuda, N., and Church, G. M. (2002). Escherichia coli gene expression responsive to levels of the response regulator EvgA. J. Bacteriol. 184, 6225-6234. doi: 10.1128/JB.184.22.6225-6234.2002

Masuda, N., and Church, G. M. (2003). Regulatory network of acid resistance genes in Escherichia coli. Mol. Microbiol. 48, 699-712. doi: 10.1046/j.1365-2958.2003. 03477.x

Mattock, E., and Blocker, A. J. (2017). How do the virulence factors of Shigella work together to cause disease. Front. Cell. Infect. Microbiol. 7:64. doi: 10.3389/fcimb. 2017.00064

Navarre, W. W., Halsey, T. A., Walthers, D., Frye, J., McClelland, M., and Potter, J. L. (2005). Co-regulation of Salmonella enterica genes required for virulence and resistance to antimicrobial peptides by SlyA and PhoP/PhoQ. Mol. Microbiol. 56, 492-508. doi: 10.1111/j.1365-2958.2005. 04553.x

Roper, D. I., Fawcett, T., and Cooper, R. A. (1993). The Escherichia coli C homoprotocatechuate degradative operon: $h p c$ gene order, direction of transcription and control of expression. Mol. Gen. Genet. 237, 241-250. doi: 10.1007/BF00282806

Shi, Y., Latifi, T., Cromie, M. J., and Groisman, E. A. (2004). Transcriptional control of the antimicrobial peptide resistance $u g t L$ gene by the Salmonella PhoP and SlyA regulatory proteins. J. Biol. Chem. 279, 38618-38625. doi: 10.1074/jbc. M406149200

Spory, A., Bosserhoff, A., von Rhein, C., Goebel, W., and Ludwig, A. (2002). Differential regulation of multiple proteins of Escherichia coli and Salmonella enterica serovar Typhimurium by the transcriptional regulator SlyA. J. Bacteriol. 184, 3549-3559. doi: 10.1128/JB.184.13.3549-3559. 2002

Srikumar, R., Kon, T., Gotoh, N., and Poole, K. (1998). Expression of Pseudomonas aeruginosa multidrug efflux pumps MexA-MexB-OprM and MexCMexD-OprJ in a multidrug-sensitive Escherichia coli strain. Antimicrob. Agents Chemother. $42,65-71$.
Stapleton, M. R., Norte, V. A., Read, R. C., and Green, J. (2002). Interaction of the Salmonella typhimurium transcription and virulence factor SlyA with target DNA and identification of members of the SlyA regulon. J. Biol. Chem. 277, 17630-17637. doi: 10.1074/jbc.M110178200

Thomson, N. R., Cox, A., Bycroft, B. W., Stewart, G. S., Williams, P., and Salmond, G. P. (1997). The rap and hor proteins of Erwinia, Serratia and Yersinia: a novel subgroup in a growing superfamily of proteins regulating diverse physiological processes in bacterial pathogens. Mol. Microbiol. 26, 531-544. doi: 10.1046/j. 1365-2958.1997.5981976.x

Tramonti, A., De Canio, M., Bossa, F., and De Biase, D. (2003). Stability and oligomerization of recombinant GadX, a transcriptional activator of the Escherichia coli glutamate decarboxylase system. Biochim. Biophys. Acta. 1647, 376-380. doi: 10.1016/S1570-9639(03)00098-0

Tramonti, A., Visca, P., De Canio, M., Falconi, M., and De Biase, D. (2002). Functional characterization and regulation of $\operatorname{gadX}$, a gene encoding an AraC/XylS-like transcriptional activator of the Escherichia coli glutamic acid decarboxylase system. J. Bacteriol. 184, 2603-2613. doi: 10.1128/JB.184.10. 2603-2613.2002

Tucker, D. L., Tucker, N., Ma, Z., Foster, J. W., Miranda, R. L., and Cohen, P. S. et al. (2003). Genes of the GadX-GadW regulon in Escherichia coli. J. Bacteriol. 185, 3190-3201. doi: 10.1128/JB.185.10.3190-3201.2003

Weatherspoon-Griffin, N., and Wing, H. J. (2016). Characterization of SlyA in Shigella flexneri identifies a novel role in virulence. Infect. Immun. 84, $1073-$ 1082. doi: 10.1128/IAI.00806-15

Yu, K., Wang, Z., Zhou, F., Jiang, J., Liu, Y., and Hu, M. et al. (2017). Quantitative analysis of Shigella flexneri protein expression under acid stress. Proteomics 17:1600381. doi: 10.1002/pmic.201600381

Zwir, I., Shin, D., Kato, A., Nishino, K., Latifi, T., and Solomon, F. et al. (2005). Dissecting the PhoP regulatory network of Escherichia coli and Salmonella enterica. Proc. Natl. Acad. Sci. U.S.A. 102, 2862-2867. doi: 10.1073/pnas. 0408238102

Conflict of Interest Statement: The authors declare that the research was conducted in the absence of any commercial or financial relationships that could be construed as a potential conflict of interest.

Copyright (c) 2018 Zhang, Ran, Wu, Li, Jiang, Wang, Cheng, Fu and Liu. This is an open-access article distributed under the terms of the Creative Commons Attribution License (CC BY). The use, distribution or reproduction in other forums is permitted, provided the original author(s) and the copyright owner(s) are credited and that the original publication in this journal is cited, in accordance with accepted academic practice. No use, distribution or reproduction is permitted which does not comply with these terms. 